\title{
Dietary lipid-dependent regulation of de novo lipogenesis and lipid partitioning by ketogenic essential amino acids in mice
}

\author{
N Nishikata, N Shikata, Y Kimura and Y Noguchi \\ Institute for Innovation, Ajinomoto Co. Inc., Kawasaki, Japan
}

Background: We have previously reported that dietary ketogenic amino acids (KAAs) modulate hepatic de novo lipogenesis (DNL) and prevent hepatic steatosis in mice. However, the dependence of the metabolic phenotypes generated by KAA on the type of dietary lipid source remains unclear.

Objective: The aim of this study was to assess the effect of KAA combined with different dietary lipid sources on hepatic DNL and tissue lipid partitioning in mice.

Design: We compared three different KAA-supplemented diets, in which a portion of the dietary protein was replaced by five major essential amino acids (Leu, Ile, Val, Lys and Thr) in high-fat diets based on palm oil (PO), high-oleic safflower oil (FO) or soy oil (SO). To compare the effects of these diets in C57B6 mice, the differential regulation of DNL and dietary lipid partitioning due to KAA was assessed using stable isotopic flux analysis.

Results: The different dietary oils showed strikingly different patterns of lipid partitioning and accumulation in tissues. High-PO diets increased both hepatic and adipose triglycerides (TG), whereas high-FO and high-SO diets increased hepatic and adipose TG, respectively. Stable isotopic flux analysis revealed high rates of hepatic DNL in high-PO and high-FO diets, whereas it was reduced in the high-SO diet. KAA supplementation in high-PO and high-FO diets reduced hepatic TG by reducing the DNL of palmitate and the accumulation of dietary oleate. However, KAA supplementation in the high-SO diet failed to reduce hepatic DNL and TG. Interestingly, KAA reduced SO-induced accumulation of hepatic linoleate and enhanced SO-induced accumulation of dietary oleate.

Conclusions: Overall, the reduction of hepatic TG by KAA is dependent on dietary lipid sources and occurs through the modulation of DNL and altered partitioning of dietary lipids. The current results provide further insight into the underlying mechanisms of hepatic lipid reduction by amino acids.

Nutrition and Diabetes (2011) 1, e5; doi:10.1038/nutd.2011.1; published online 28 March 2011

Keywords: ketogenic amino acids; hepatic steatosis; metabolic flux analysis

\section{Introduction}

The rising prevalence of obesity - a major health concern worldwide - has been linked to a number of cardiovascular and metabolic disorders, such as hypertension, insulin resistance, hyperlipidemia and, more recently, non-alcoholic fatty liver disease, also known as hepatic steatosis. ${ }^{1,2}$ Physical inactivity, dietary behavior and inherited predisposition are the predominant causes of excessive fat accumulation in

Correspondence: Dr Y Noguchi, Institute for Innovation, Ajinomoto Co. Inc., 1-1 Suzuki-Cho, Kawasaki-ku, Kawasaki 210-8681, Japan.

E-mail: yasushi_noguchi@ajinomoto.com

Received 29 September 2010; revised 7 January 2011; accepted 16 February 2011 adipose and non-adipose tissues, which subsequently leads to metabolic disorders. In diets designed to prevent and treat such excessive fat accumulation by manipulating energy content, macronutrient distribution has been examined by controlling the proportions of fat, carbohydrate and protein, commonly with reductions in dietary fat. ${ }^{3,4}$ Although this approach appears to be effective for reducing energy density and increasing the loss of lipids in adipose tissue in the short term, ${ }^{3,4}$ increased dietary carbohydrate levels activate de novo lipogenesis (DNL) to compensate for the lower fat intake and then induce lipid accumulation in non-adipose tissues in the long term. ${ }^{5,6}$ Likewise, a low-carbohydrate diet has been shown to be an effective approach for body fat reduction in the short term, but the long-term effects have not been established. ${ }^{7}$ Recent studies in humans show that 
a high-protein diet may also have beneficial effects on body fat maintenance by inducing satiety or reducing hepatic lipid deposition. ${ }^{8}$

In addition to macronutrient proportions, specific macronutrient elements such as sugars, ${ }^{9}$ fatty acids ${ }^{10}$ and amino acids ${ }^{11,12}$ are important factors in the regulation and dysregulation of lipid metabolism. For example, the difference between monounsaturated fatty acids (MUFAs) and saturated fatty acids (SFAs) has been well characterized; ${ }^{13-15}$ these two types of fatty acid affect postprandial lipid metabolism differently. ${ }^{16,17}$ In addition, polyunsaturated fatty acid (PUFA) supplementation has been reported to prevent insulin resistance ${ }^{18,19}$ and hepatic steatosis. ${ }^{20,21}$ Several reports suggest that dietary medium-chain fatty acids promote energy expenditure and fatty acid oxidation and improve lipid metabolism in rats ${ }^{22}$ and humans ${ }^{23}$ compared with long-chain fatty acids.

Previous studies have shown that dietary withdrawal of the essential amino acids lysine or threonine induces severe hepatic steatosis in rodents. ${ }^{11,12}$ Furthermore, a role for the amino-acid deprivation sensor GCN2 in regulating hepatic lipid homeostasis has recently been revealed. ${ }^{24,25}$ Leucine and lysine, which are both ketogenic amino acids (KAAs), have unique metabolic characteristics and produce various short-chain fatty acyl-CoAs, such as isovalaryl-CoA, 3-hydroxy-3-methylglutaryl-CoA and glutaryl-CoA, through catabolism. In addition to leucine and lysine, isoleucine, valine and threonine also produce short, branched-chain acyl-CoAs. $^{26} \mathrm{~A}$ few studies have reported the potential importance of these branched-chain acyl-CoAs in regulating lipid homeostasis through transcription factors or nuclear receptors. ${ }^{27,28}$ We previously reported that the supplementation of KAA that generate acyl-CoA rescued palmitateinduced hepatic lipoapoptosis in vitro ${ }^{29}$ and also prevented hepatic steatosis through the repression of DNL in mice. ${ }^{30}$ However, the underlying mechanisms and effects of KAA versus dietary lipid species on these phenotypes have yet to be elucidated.

In this study, we examined KAA supplementation in C57B6 mice fed either a regular high-lard diet or one of three different high-fat diets (HFDs), that is, SFA-rich palm oil (PO), MUFA-rich high-oleic safflower oil (FO) and PUFArich soy oil (SO) diets. Using gas chromatography-mass spectrometry (GC-MS)-based lipid profiling and stable isotopic metabolic flux analysis, we assessed the dependence of the effect of KAA on the type of dietary lipid in terms of both the regulation of hepatic DNL and the dietary lipid partitioning to tissues.

\section{Materials and methods}

Animals

All studies were reviewed and approved by the Animal Care Committee of Ajinomoto Co. Inc. Ten-week-old male C57B6 mice and $o b / o b$ mice, were obtained from Charles River Laboratory, Japan Inc. (Yokohama, Japan). All mice were housed in colony cages and maintained on a 12:12-h light and dark rhythm with free access to water. After $3 \mathrm{~h}$ of fasting, blood was collected on ice in tubes containing EDTA (NONCLOT-D, Daiichi Pure Chemicals, Tokyo, Japan). Liver, epididimal fat and gastrocnemius muscle samples were collected for lipid and gene expression analysis. All collected tissues were snap-frozen in liquid nitrogen and stored at $-80^{\circ} \mathrm{C}$.

\section{Diets}

As documented in our previous report, we manipulated dietary amino-acid composition by replacing part of the protein content with a free amino-acid mixture in either a control diet or a high-KAA diet. ${ }^{30}$ Supplementary Tables S1 and S2 show the composition of the diets used in this study. The casein-mimic-free amino-acid mixture used for the control diet contained the following components: $2.5 \%$ His, $4.5 \%$ Phe, $8.8 \%$ Lys-HCl, 1.1\% Trp, 3.8\% Thr, 2.4\% Thr, 2.4\% Met, 4.5\% Ile, 5.7\% Val, 8.1\% Leu, 9.4\% Pro, 9.4\% Asn $\mathrm{H}_{2} \mathrm{O}, 4.9 \%$ Tyr, $2.6 \%$ Ala, 3.3\% Arg, 5.1\% Ser, 9.2\% Glu, 9.2\% Gln, $1.6 \%$ Gly, $0.5 \%$ cysteine, $3.2 \%$ Asp and $6.2 \%$ starch. On the basis of the partial protein replacement by free KAA, the essential amino acid/non-essential amino acid ratio in the diet was fortified from the control value of 0.8 (for casein) to 1.76. To avoid differences in the amounts of dietary total amino acids, fat and carbohydrates among the diets, the total amino acids, including protein-amino acids, were equalized, if necessary, using casein-mimic-free amino-acid mixture to provide $20 \%$ of dietary calories. On the basis of this protocol, we prepared the high-lard diet and the three different types of HFDs consisting of PO, FO and SO. As a percentage of total energy, the diets consisted of $26.4 \%$ carbohydrate, $52.6 \%$ fat and $20 \%$ protein plus amino acids. The high-KAA diet contained $13.1 \%$ basal casein, with $6.9 \%$ consisting of a selected KAA mixture of leucine, isoleucine, valine, lysine and threonine in the proportions shown in Supplementary Tables S1 and S2.

\section{Blood biochemistry}

Blood glucose, cholesterol and triglycerides (TG) were measured using DRI-CHEM5500 (Fujifilm, Tokyo, Japan). Plasma-free fatty acids were determined using an enzymatic method with an automated kit according to the manufacturer's specifications (Wako Pure Chemical Industries, Osaka, Japan). Serum leptin and insulin were determined using commercial mouse ELISA kits (Seikagaku-kogyo Co., Tokyo, Japan).

\section{Energy expenditure}

The energy expenditure of individual mice was measured using indirect calorimetry. An animal was housed in a metabolic cage for $24 \mathrm{~h}$, allowing for the separate collection 
of urine and feces. ${ }^{28}$ Food was available between 1900 and 0800 hours. Oxygen consumption and $\mathrm{CO}_{2}$ production were determined every $5 \mathrm{~min}$ in an open chamber with the mass spectrometry-based $\mathrm{O}_{2}$ and $\mathrm{CO}_{2}$ analyzer ARCO-2000 (ARCO system, Chiba, Japan). Oxygen consumption was normalized by lean body mass.

\section{Tissue lipid extraction}

Liver lipid extraction was performed using isopropanol and hexane based on a modification of a method described previously. $^{31}$ This procedure extracts a mixture of TG, diglycerides, mono-glycerides, cholesterol, fatty acids and other polar lipids. After extraction, fractionation was performed to collect each lipid class. In brief, $150 \mathrm{mg}$ of liver or $40 \mathrm{mg}$ of epididymal fat tissue was homogenized in $1.5 \mathrm{ml}$ of isopropanol and then sonicated for $30 \mathrm{~min}$ after the addition of $3 \mathrm{ml}$ hexane. As an internal standard, $0.1 \mathrm{mg}$ triheptadecanoic acid was added to each sample. The homogenate was incubated overnight for further extraction. After the addition of $1 \mathrm{ml}$ saline, the mixture was centrifuged at 3500 r.p.m. for $15 \mathrm{~min}$, and the supernatant was collected into new tubes.

Because the extracted solvent contains a mixture of different classes of lipids, fractionation was performed to efficiently analyze each class of lipids using a silica gel column together with gradual elution with solvents of increasing polarity as described in a previous report. ${ }^{32}$ The extracted solvent was evaporated to dryness, resolved in $500 \mu \mathrm{l}$ of isooctane/ethyl acetate (10:1) and applied to a preequiberated silica gel column (InertSep SI, GL Sciences, Tokyo, Japan). The column was then eluted with $4 \mathrm{ml}$ isooctane/ethyl acetate (10:1). This fraction contained cholesterol esters and TG. This process was repeated with isooctane/ethyl acetate (3:1) and methanol, and each elutant contained free fatty acids, diglycerides and polar lipids.

\section{Lipid analysis by GC-MS}

To analyze the fatty acid profile of TG, the triglyceride fraction was saponified with $0.5 \mathrm{~N} \mathrm{NaOH}$ dissolved in methanol, and methyl was esterized with boron trifluoride as described previously. ${ }^{29}$ The fatty acid methyl esters were analyzed using electron impact ionization by GC-MS on an Agilent 5975C model coupled with a 7890A model gas chromatograph (Agilent, Santa Clara, CA, USA). A DB-225 ms capillary column $(30 \mathrm{~m} \times 0.25 \mathrm{~mm}$ film $0.25 \mu \mathrm{m}$, Agilent) was used in all lipid analyses. The initial oven temperature was $190^{\circ} \mathrm{C}$, which was then increased by $3{ }^{\circ} \mathrm{Cmin}^{-1}$ to $230^{\circ} \mathrm{C}$ and held for $2 \mathrm{~min}$. The Mass Selective Detector (MSD) transfer line was set at $280^{\circ} \mathrm{C}$. The components of the sample were repetitively scanned from 50 to $550 \mathrm{~m} / \mathrm{z}$. The MS quad was $150^{\circ} \mathrm{C}$, and the MS source was set at $230{ }^{\circ} \mathrm{C}$.

Peak enumeration and spectral deconvolution was performed by AMDIS software (http://chemdata.nist.gov/mass-spc/amdis/; National Institute of Standards and Technology). The resulting
ELU files (output from AMDIS) were further analyzed by SpectConnect software (http://spectconnect.mit.edu/) to identify well-conserved peaks among multiple GC-MS chromatograms. ${ }^{33}$

\section{Lipogenic flux estimation}

Two weeks before killing, mice were given an intraperitoneal injection of deuterated water $\left(\mathrm{D}_{2} \mathrm{O}\right)$ equal to $4 \%$ of their body weight in saline at 1000 hours. Animals were then maintained on drinking water containing $6 \% \mathrm{D}_{2} \mathrm{O}$ for 2 weeks. This procedure was designed to maintain deuterium enrichment in the body water at around 3-4\% throughout the study. Animals were fasted for $3 \mathrm{~h}$ before killing.

Using $\mathrm{D}_{2} \mathrm{O}$ and mass isotopomer analysis, the rates of synthesis can be calculated from the ratio of isotope-labeled fatty acids to unlabeled fatty acids. ${ }^{34,35}$ The major peaks in the ion chromatogram of fatty acids in the liver are the longchain fatty acids palmitate (16:0), stearate (18:0) and oleate (18:1). The fractional synthesis rates of these three fatty acids were calculated. The chain elongation flux of palmitate to stearate was determined by dividing the amount of newly synthesized C18:0 by that of newly synthesized 16:0. The desaturation flux of stearate to oleate was calculated by dividing the amount of newly synthesized $18: 1$ by that of newly synthesized 18:0. The deuterium enrichment of body water was determined by isotopic exchange with $\left[\mathrm{U}_{-}{ }^{13} \mathrm{C}_{3}\right]$ acetone as described previously. ${ }^{36}$

\section{Real-time PCR}

Total RNA was extracted from homogenized liver using an RNeasy kit (Qiagen, Germantown, MD, USA) following the manufacturer's instructions. Messenger RNA was then extracted from total RNA preparations using an Oligotex kit (Qiagen) following the manufacturer's instructions. The quality and integrity of the RNA were checked using the $\mathrm{A}_{260} / 280$ ratio and electrophoresis on a formaldehyde/agarose gel, respectively. Equal amounts of RNA were reverse transcribed using Superscript II reverse transcriptase (Invitrogen, Carlsbad, CA, USA) as per the manufacturer's instructions. Primers for reverse transcriptase-PCR were designed using the primer design software Primer3, after which the sequence homology for related proteins was checked. Specifically, $18 \mathrm{~S}$ ribosomal RNA primers were used as an endogenous control. Reverse transcriptase-PCR was performed on an ABI Prism 7700 Sequence Detection System (PE Applied Biosystems, Foster, CA, USA), and the data obtained were analyzed using the provided software. The reaction mixture consisted of $4 \mu \mathrm{l}$ complementary DNA template, $10 \mu 1$ Sybr Green PCR master mix (Roche Biochemicals, Indianapolis, IN, USA), $2 \mu \mathrm{l} 0.25-1 \mu \mathrm{M}$ forward primer and $2 \mu \mathrm{l} 0.25-1 \mu \mathrm{M}$ reverse primer in a $20 \mu \mathrm{l}$ reaction volume. The PCR protocol consisted of a single $10 \mathrm{~min}$ denaturation cycle at $95^{\circ} \mathrm{C}$ followed by 40 cycles of denaturation at $95^{\circ} \mathrm{C}$ for $15 \mathrm{~s}$ and annealing/extension at $60^{\circ} \mathrm{C}$ for $1 \mathrm{~min}$. Standard curves for each gene and endogenous $18 \mathrm{~S}$ ribosomal RNA 
control were obtained. The efficiency of PCR amplification was $100 \%$, and the $R^{2}$-value was between 0.995 and 0.999 . All reverse transcriptase-PCR data were expressed as relative mRNA levels after normalization to 18S ribosomal RNA. Primer pair sequences are listed in Supplementary Table S3.

\section{Statistics}

Data are presented as the means \pm s.e.m. unless otherwise indicated. Data were analyzed by one-way analysis of variance using Tukey's post hoc test to determine statistical significance for all pairwise multiple comparison procedures and Dunnett's test for multiple comparisons against the control group.

\section{Results}

\section{Effect of KAA on obese mice models}

We first examined KAA fortification in C57B6 mice fed either the low-fat diet (LFD) or the lard-based HFD. Before the experiment, mice were fed either LFD or HFD for 2 months (pre-LFD or pre-HFD). At day 0, the average body weights of mice in the pre-LFD and -HFD groups were 31.6 \pm 0.3 and $37.1 \pm 0.5$, respectively. Under pre-LFD conditions, the 4 weeks of high-KAA diets significantly reduced body weight gain (BWG) in both LFD and HFD (Figure 1a right panel and Figure 1b). In pre-HFD conditions, however, the high-KAA diet reduced BWG only in LFD, but no clear reduction was seen in HFD. Table 1 summarizes weights of the liver, kidney, gastrocnemius and soleus muscle as well as the different part of adipose tissues at the end of experiment. No significant changes in these tissue weights were observed, except for adipose. During the experimental period, KAA fortification of the HFD had no effect on food intake, but reduced the amount of food intake on the LFD. KAA fortification of the HFD increased oxygen consumption without changes in the respiratory quotient compared with the control HFD (Figures $1 \mathrm{~d}$ and e).

Under pre-LFD conditions, the high-KAA diets significantly reduced postprandial insulin and fat accumulation for both the LFD and the HFD. The pre-HFD, high-KAA diets, however, reduced those two parameters only under the LFD condition, but had no effect under HFD conditions (Figure 2a). Unlike plasma insulin and fat accumulation, the high-KAA diets reduced hepatic fat contents in all conditions, with more significant reductions under HFD conditions than under LFD conditions (Figure 2a, bottom). To further understand the associations between postprandial insulin and both hepatic and adipose fat accumulations, we examined dietary KAA fortification in hyperinsulinemic $o b / o b$ mice. The $o b / o b$ mice were fed an HFD or LFD for 2 weeks with or without dietary KAA fortification. In $o b / o b$ mice, the high-KAA diet reduced postprandial insulin only in the LFD (Figure 2b). The KAA diets significantly reduced hepatic fat conditions under both the LFD and the HFD. Similarly, in C57B6 mice under HFD conditions, the reduction due to the high-KAA diet was more significant in improving hepatic steatosis than under the LFD (Figures $2 b$ and c). The dietary KAA fortification did not affect body fat content in $o b / o b$ mice after at least 2 weeks of feeding (Figure 2b).

\section{Effect of KAA on varying dietary lipid-induced metabolic phenotypes}

These metabolic changes under dietary KAA fortification in both C57B6 and ob/ob mice suggest that KAA fortification predominately affects hepatic fat rather than adipose fat contents, and it is more effective under high-fat conditions. To address the relationship between dietary KAA and fatty types, we next compared three different KAA-supplemented HFDs, namely, PO-, FO- or SO-based diets. The control LFD was prepared by blending the three oils.

Dietary lipids of these three different oils were analyzed by GC-MS to confirm their fatty acid compositions (Figure 3a). More than half of the fatty acids in the PO were SFA, of which nearly 50\% were palmitate (16:0) and 5\% were stearate (18:0). The FO was mostly MUFA, of which over $80 \%$ was oleate. The SO consisted primarily of PUFA, of which $52 \%$ was linoleate $(18: 2)$ and $6 \%$ was linolenate (18:3).

Table 2 illustrates the postprandial variables at the end of the 8-week experimental period. The high-KAA diet significantly reduced postprandial plasma cholesterol in the highPO and high-FO conditions, whereas no clear reduction was observed in mice consuming the high-SO diet. Although HFD feeding caused only slight increases in blood glucose and TG in comparison to the LFD group, the high-KAA diet almost normalized these values in mice consuming the HFDs (Table 2). Under these conditions, no significant changes in food intake were observed between control, high-fat and KAA fortified groups (Figure 3b). BWG due to high-PO or high-SO feeding was significantly repressed by KAA fortification, whereas no significant increase in BWG on high-FO diets or repression by KAA feeding was observed (Figure 3c). At the end of the 8-week experimental period, the epididymal fat weight decreased by $34 \%$ in the KAA group as compared with both the control PO and the SO groups (Figure 4c).

\section{Effect of KAA on hepatic lipid contents with varying dietary lipids}

As compared with the level in the control LFD group, hepatic TG content was increased by 75 and 29\% under the high-PO and high-FO diets, respectively, whereas no significant increase was observed under the high-SO diet (Figure 4c). Similar trends were observed regarding hepatic cholesterol content, which increased by 26 and $43 \%$ under the high-PO and -FO diets. However, KAA fortification of both high-PO and -FO diets repressed increases in both hepatic TG and cholesterol. There were no clear changes in hepatic TG and 
a

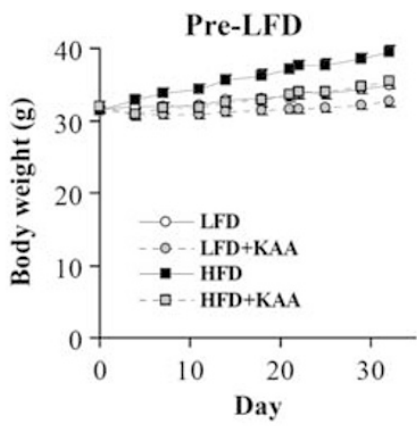

b
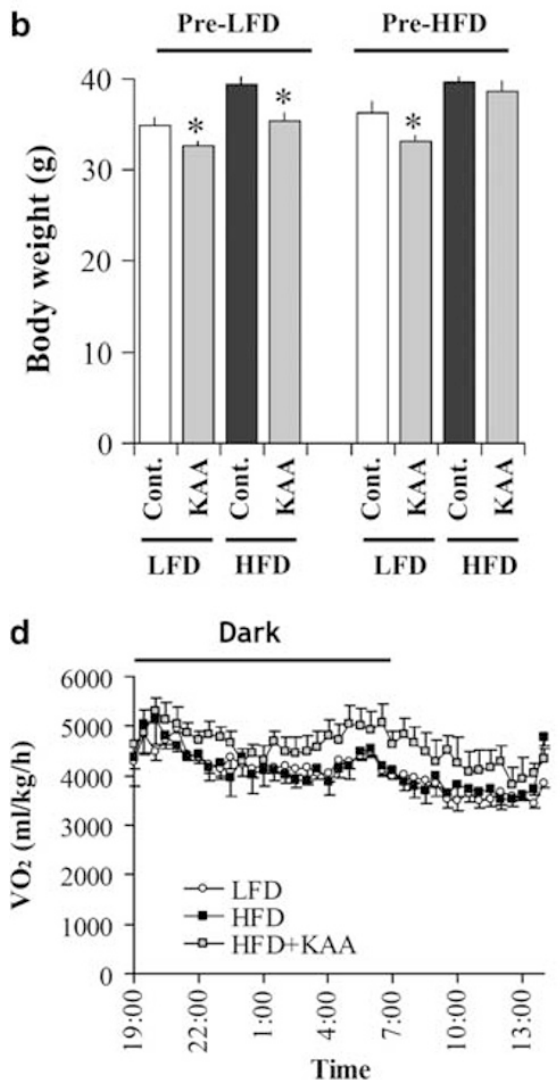

\section{Pre-HFD}

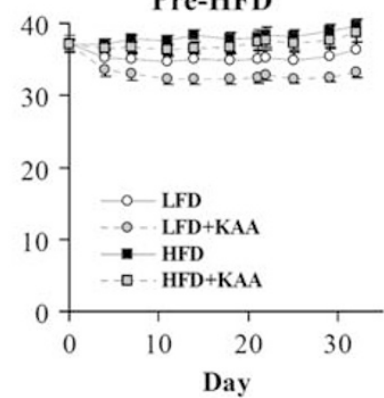

C

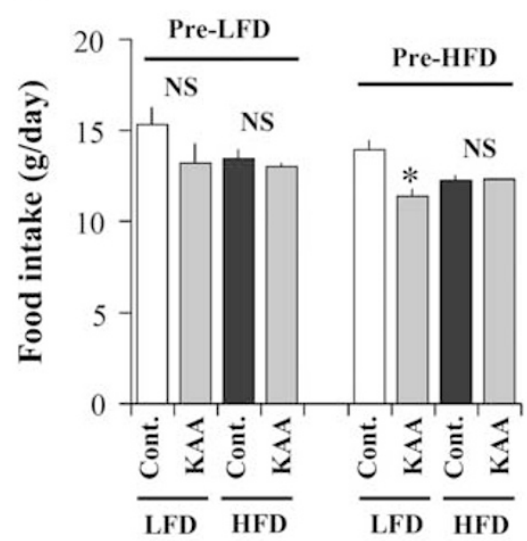

e

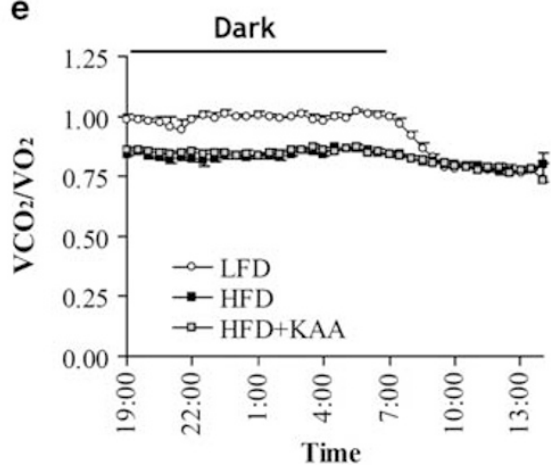

Figure 1 Effects of high-KAA diets on diet-induced obesity. Growth curves (a), body weights (b), average food intake (c), oxygen consumption (d), and respiratory quotient (e) of C57B6 mice fed for 4 weeks with or without KAA fortification under LFD and HFD conditions (mean \pm s.e.m., $n=9$ for each group). KAA fortifications were started after 2 months of pretreatment of LFD or HFD (shown as pre-LFD or pre-HFD). At day 0 , the average body weights of mice under pre-LFD and pre-HFD conditions were $31.6 \pm 0.3$ and $37.1 \pm 0.5$, respectively. For oxygen consumption and respiratory quotients, values were obtained over 3 days following a week of acclimatization to the metabolic chamber, and only the results from the HFD with or without KAA fortification are shown. ${ }^{*} P<0.05$ for all high-KAA groups as compared with the control LFD or HFD groups. NS, not significant.

cholesterol contents in high-SO groups compared with the control LFD group (Figure 4c).

\section{De novo lipogenesis and lipid partitioning}

To understand the mechanism of TG regulation under the high-KAA condition, metabolic flux analysis was used to quantify changes in DNL and non-DNL lipid partitioning to tissues. Figure 5 summarizes the different fatty acid sources contributing to hepatic and adipose TG in C57B6 mice fed various diets for 8 weeks.

In fat tissue, the contribution of DNL to palmitate and oleate contents was generally smaller and lower in all HFD groups as compared with the control LFD group (Figure 5, right panels). In contrast, a large contribution of DNL to palmitate and oleate synthesis in the liver was observed 
Table 1 Summary of tissue weights in C57B6 mice fed varying diets

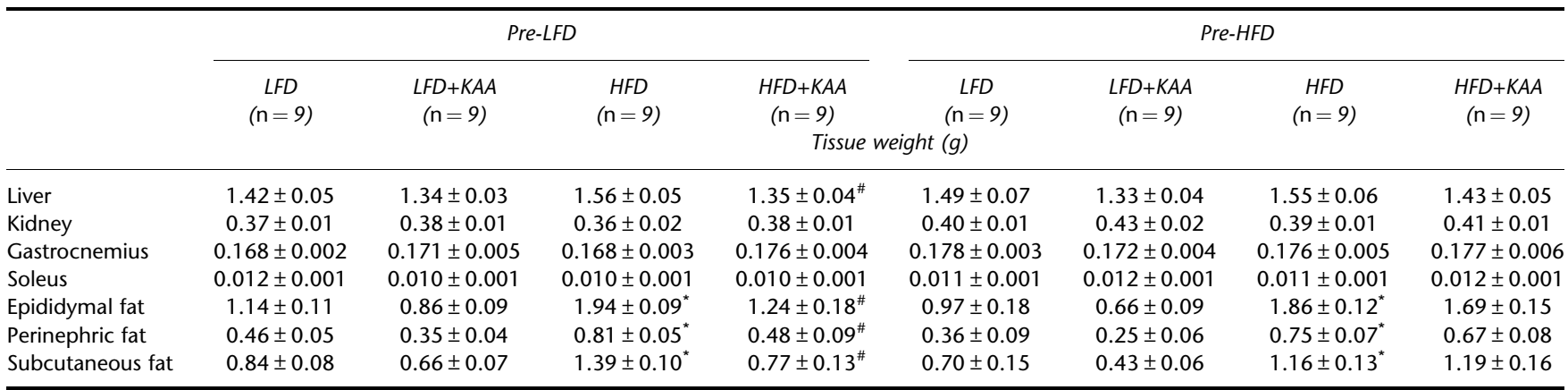

Abbreviations: HFD, high-fat diet; KAA, ketogenic amino acid; LFD, low-fat diet. Data represent mean \pm s.e.m. ${ }^{*} P<0.05$ for all high-fat groups compared with control LFD group; ${ }^{\#} P<0.05$ for high-KAA group with control HFD.

a
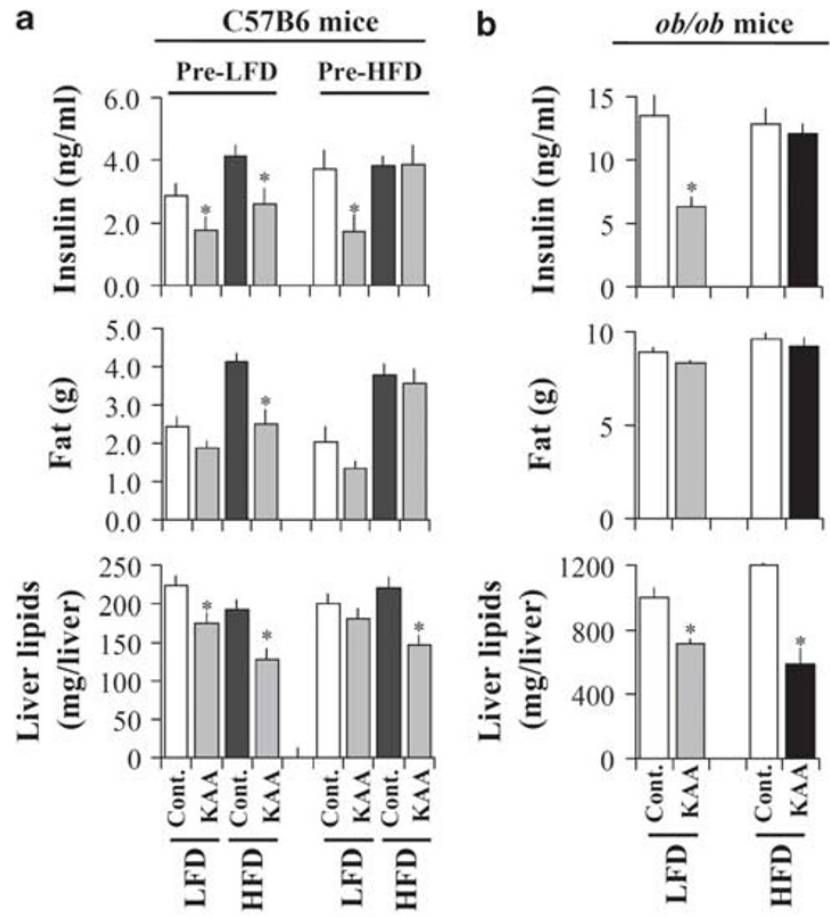

C $o b / o b$ mice

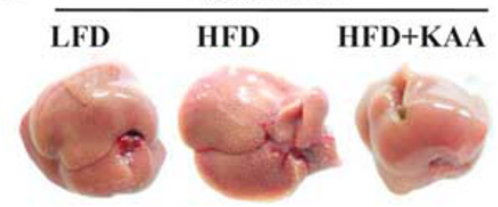

Figure 2 Effects of high-KAA diets on adipose and hepatic fat contents. (a) Plasma insulin ( $3 \mathrm{~h}$ fast), fat mass (subcutaneous, epididymal and perinephric) and total liver lipids in pre-LFD- and pre-HFD-treated mice fed the indicated diet for another 4 weeks $(n=9)$. (b) Plasma insulin ( $3 \mathrm{~h}$ fast), fat mass and total liver lipids in $o b / o b$ mice fed LFD and HFD with or without KAA fortification for 2 weeks $(n=6)$. (c) Representative liver pictures from $o b / o b$ mice fed LFD, HFD and HFD with KAA fortification for 2 weeks. All values are expressed as means \pm s.e.m. $(n=6)$. ${ }^{*} P<0.05$ for high-KAA diet compared with the corresponding control group. under both high-PO and high-FO diets and in the control LFD group; this effect was significantly reduced in mice consuming the high-SO diet (Figure 5, left and right panels). The different dietary oils also showed different patterns of non-DNL partitioning and accumulation in both tissues, presumably from different dietary fatty acids. Particularly in the liver, high levels of PO, FO and SO increased non-DNL palmitate, oleate and linoleate, respectively.

KAA supplementation in high-PO and high-FO diets significantly reduced the DNL of palmitate and oleate and the non-DNL partitioning of these two fatty acids into the liver (Figure 5, left panels). KAA supplementation under the high-SO condition, however, failed to reduce the hepatic DNL and TG. Interestingly, KAA significantly reduced SO-induced accumulation of linoleate, but increased the non-DNL oleate in both tissues (Figure 5). In contrast, KAA reduced FO-induced non-DNL oleate, but increased linoleate (Figure 5).

Changes in hepatic lipogenic gene expression by dietary fat and amino acids

Expression of hepatic genes involved in lipogenesis was measured in response to varying HFD feeding with or without KAA supplementation (Figure 6). The high-KAA diet repressed the high-PO induced increase in the sterol regulatory element-binding protein-1c (SREBP-1c), but failed to repress expression under high-FO and high-SO diets. Expression of adenosine triphosphate citrate lyase $(A C L)$, acetyl-CoA carboxylase $(A C C)$, stearoyl-CoA reductase (SCD-1) and Elovl-6 was reduced under all three HFDs as compared with the control LFD group, whereas KAA supplementation additively repressed the expression of these enzymes, particularly in both high-PO and high-FO diets (Figure 6). The high-SO diet dramatically decreased $A C L$, $A C C$ and SCD-1 expression by $70-90 \%$ as compared with the LFD group; no additional effect due to KAA was observed. Fatty acid synthase (FAS) expression was reduced in all HFDs, 
and no clear effect of KAA supplementation was observed (Figure 6). Regarding glucolytic genes, only the high-PO diet increased the expression of glucokinase $(G K)$ genes, and KAA repressed such expression. In addition, no clear changes due to KAA were observed in liver pyruvate kinase.
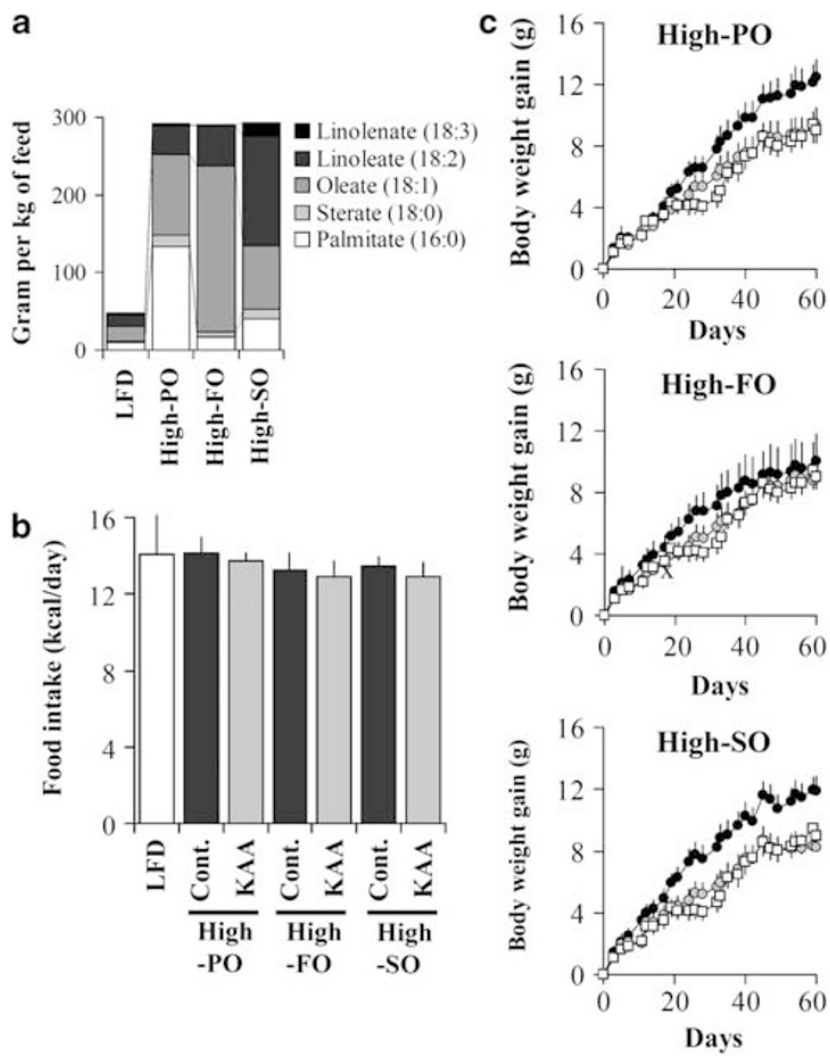

Figure 3 Body weight gain of C57B6 mice fed different high-fat diets. (a) Fatty acid contents in each diet were estimated based on nutritional composition (Supplementary Table S2) and fatty acid composition in palm oil (PO), safflower oil (FO) and soy oil (SO). Fatty acids were determined using GC-MS as described in Materials and methods section. (b, c) Food intake and body weight gain of C57B6 mice fed for 8 weeks with control low-fat diet (LFD), high-palm oil (high-PO), high-safflower oil (high-FO) or high-soy oil (high-SO) diets with or without KAA fortification was compared. In each panel, the white square, black circle and gray circle represent low-fat diet, control high-fat and KAA fortified high-fat diets, respectively. Average body weight at day 0 was $27.2 \pm 0.25$. All values are expressed as means \pm s.e.m. ( $n=6$ for each group).
Correlation of lipogenic fluxes and gene expression

To understand the regulatory differences of lipogenic genes induced by dietary lipid and amino acids, we performed correlation analyses of lipogenic fluxes and gene expression patterns. In this study, we estimated elongase and desaturase fluxes in addition to palmitate synthesis (DNL of palmitate), as shown in Figure 5, and then we compared these fluxes to the expression of the lipogenic genes $A C L, A C C, F A S$, Elovl-6 and $S C D$-1. Figure 7 shows the correlation between palmitate synthetic flux and related expression patterns of $A C L, A C C$ and FAS. The flux of palmitate synthesis shows a relatively high correlation with ACL and ACC ( $r=0.53$ and 0.38 , respectively), whereas no clear correlation with $F A S$ was seen. Correlations between elongase flux and Evovl-6 expression and between desaturase flux and $S C D-1$ expression were also assessed; the desaturase reaction, but not the elongase reaction, exhibited a correlation between flux and expression patterns (Figure 7).

\section{Discussion}

This study found that dietary lipids and amino acids reciprocally affect fat accumulation in mice through the regulation of DNL and tissue lipid distribution. Increased intake of nutritional elements such as carbohydrates and fat has been reported to lead to obesity and insulin resistance. . $^{3,4}$ Several studies have suggested that dietary fatty acid composition affects the development of insulin resistance, hepatic steatosis and obesity. ${ }^{10}$ Different types of fatty acids display different metabolic phenotypes, including oxidation and deposition rate differences that lead to body fat changes. ${ }^{37}$ The chemical structure of dietary fatty acids and their chain length, degree of unsaturation, the position of double bonds and their configuration seem to affect their metabolic fate. For example, one comparison of dietary MUFA intake with SFA revealed a decrease in fat deposition under a diet rich in MUFA. ${ }^{38}$ For unsaturated long-chain fatty acids, PUFA from fish oil has been reported to decrease fat mass in rodents. ${ }^{39}$

To address these issues, we examined the effects of three different diets high in PO, FO and SO on lipid metabolism in C57B6 mice, where PO, FO and SO predominantly contained

Table 2 Summary of metabolic parameters in C57B6 mice fed varying high-fat diet with or without KAA supplementation

\begin{tabular}{|c|c|c|c|c|c|c|c|}
\hline & \multirow[b]{2}{*}{$L F D(\mathrm{n}=6)$} & \multicolumn{2}{|c|}{ High-PO } & \multicolumn{2}{|c|}{ High-FO } & \multicolumn{2}{|c|}{ High-SO } \\
\hline & & Cont. $(n=6)$ & $K A A(\mathrm{n}=6)$ & Cont. $(\mathrm{n}=6)$ & $K A A(\mathrm{n}=6)$ & Cont. $(\mathrm{n}=6)$ & $K A A(\mathrm{n}=6)$ \\
\hline Glucose $\left(\mathrm{mg} \mathrm{dl}^{-1}\right)$ & $189.5 \pm 5.3$ & $196.7 \pm 4.8$ & $169.8 \pm 9.8^{\#}$ & $209.0 \pm 7.1$ & $187.3 \pm 12.7$ & $220.7 \pm 3.1^{\star}$ & $211.3 \pm 9.2$ \\
\hline Triglyceride $\left(\mathrm{mg} \mathrm{dl}^{-1}\right)$ & $182.5 \pm 5.7$ & $198.3 \pm 4.8$ & $182.7 \pm 6.6$ & $185.4 \pm 9.7$ & $181.0 \pm 8.1$ & $198.1 \pm 5$ & $175.9 \pm 8.3 \#$ \\
\hline Cholesterol $\left(\mathrm{mg} \mathrm{dl}^{-1}\right)$ & $186.0 \pm 4.8$ & $197.5 \pm 3.7$ & $177.5 \pm 7.2 \#$ & $197.2 \pm 7.6$ & $184.2 \pm 8.4$ & $209.4 \pm 2.7^{*}$ & $193.6 \pm 8.7$ \\
\hline
\end{tabular}

Abbreviations: FO, safflower oil; HFD, high-fat diet; KAA, ketogenic amino acid; LFD, low-fat diet; PO, palm oil; SO, soy oil. Data represent mean \pm s.e.m. * $P<0.05$ for all high-fat groups compared with LFD group; ${ }^{\#} P<0.05$ for high-KAA group with control HFD. 

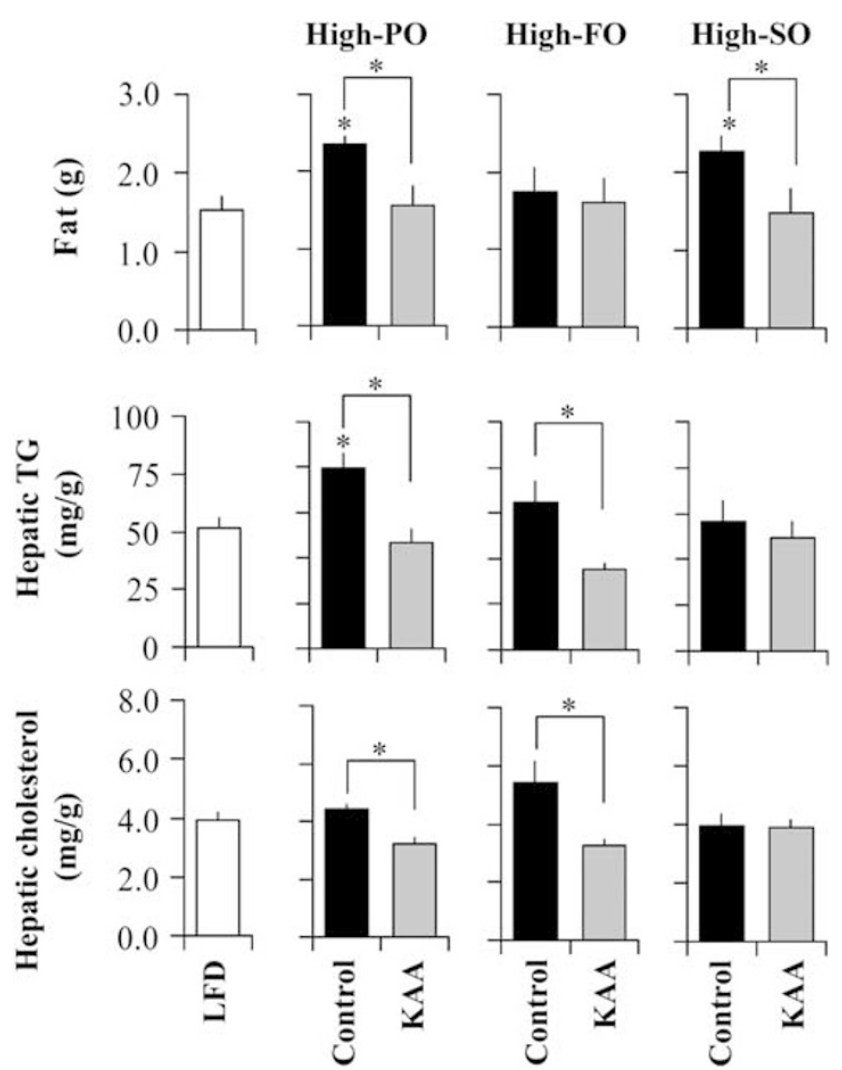

Figure 4 Fat, hepatic triglyceride and cholesterol contents in C57B6 mice fed different diets. Epididymal fat in C57B6 mice fed for 8 weeks with highpalm oil (high-PO), high-safflower oil (high-FO) or high-soy oil (high-SO) diets with or without KAA fortification was determined at the end of the study (upper panel). Liver samples were obtained from C57B6 mice fed for 8 weeks with the LFD or the varying HFD with or without KAA fortification. Hepatic TG and cholesterol were enzymatically determined as described in the Materials and methods section (middle and bottom panels). All values are expressed as means \pm s.e.m. ( $n=6$ for each group). ${ }^{*} P<0.05$ for all HFD control groups as compared with the LFD group or as indicated.

SFA, MUFA and PUFA, respectively. Our data show clear differences in fat accumulation among these dietary groups; both $\mathrm{PO}$ and $\mathrm{SO}$ (but not FO) increased BWG in mice. In addition, PO induced fat accumulation in both liver and adipose tissue, whereas FO and SO only induce it in liver and adipose tissue, respectively. These differences in fat accumulation in tissues can also be explained according to the different types of dietary fat and their behaviors in lipid metabolism. For instance, in vivo and in vitro studies have shown that PUFA, but not MUFA nor SFA, seems to suppress the expression of lipogenic genes primarily through decreased expression of SREBP-1c. ${ }^{40}$ Our data show that all three oils increase $S R E B P-1 c$ expression in mice, but strongly repress downstream genes such as $S C D-1$ and Elovl-6. The differences in the expression of SREBP-1C and other lipogenic genes based on different HFDs were not clear in this study. Both the proportions of dietary fatty acid types and the
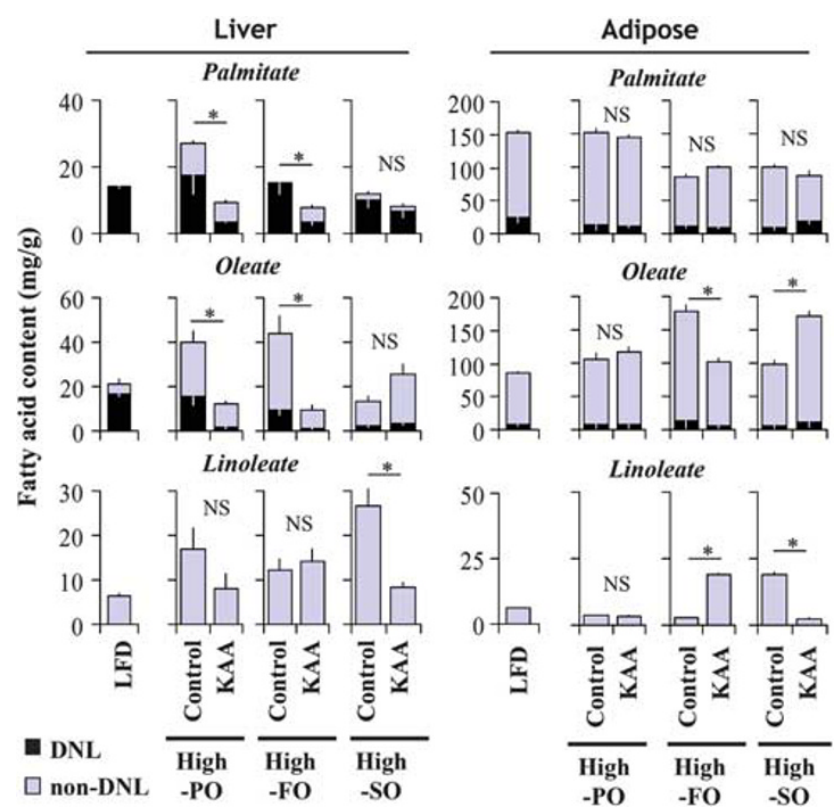

Figure 5 Fatty sources for hepatic and adipose trigylyceride in C57B6 mice fed different diets. Metabolic flux of DNL was analyzed by identifying fatty sources for hepatic (left panels) and adipose (right panels) triglycerides using deuterated water labeling and mass isotopomer distribution analysis (see Materials and methods section). The contribution of DNL to total triglyceride fatty acids in both tissues was separately estimated for palmitate (c16:0) and oleate (c18:1); only non-DNL values are shown for linoleate (18:2). Black and blue bars represent DNL and non-DNL sources, respectively. All values are expressed as means \pm s.e.m. ( $n=6$ for each group). ${ }^{\star} P<0.05$ for all HFD control groups as compared with the LFD group or as indicated.

duration of treatment may further complicate the effects on lipid metabolism.

Recent studies have shown that dietary protein could affect fat accumulation by satiating and metabolic effects. ${ }^{41}$ In addition, amino acid, fat and glucose metabolisms are highly coordinated through multiple organ systems, including the liver. ${ }^{25,42,43}$ However, the mechanism by which dietary amino acids regulate lipid metabolism is still unclear. We previously reported that the manipulation of the essential amino acid/non-essential amino acid ratio by supplementation with both the KAA lysine and threonine and the branched-chain amino acids prevented hepatic steatosis through repression of DNL in mice. ${ }^{30}$ To extend this finding, we examined the effect of KAA supplementation on the murine lipid synthetic pathway as well as on the fat deposition that occurs when mice are fed different HFDs. In this study, we replicated our previous findings on the reduction of hepatic lipid by dietary KAA in C57B6 mice, as we investigated KAA fortification under LFD and HFD in mice. Our data clearly duplicated a reduction in hepatic and body fat due to KAA, with increased oxygen consumption under HFD conditions. However, we found that KAA supplementation had relatively less of an effect under LFD conditions, although it introduced a slight anorexic effect under LFD. Therefore, we hypothesized that the reduction of 


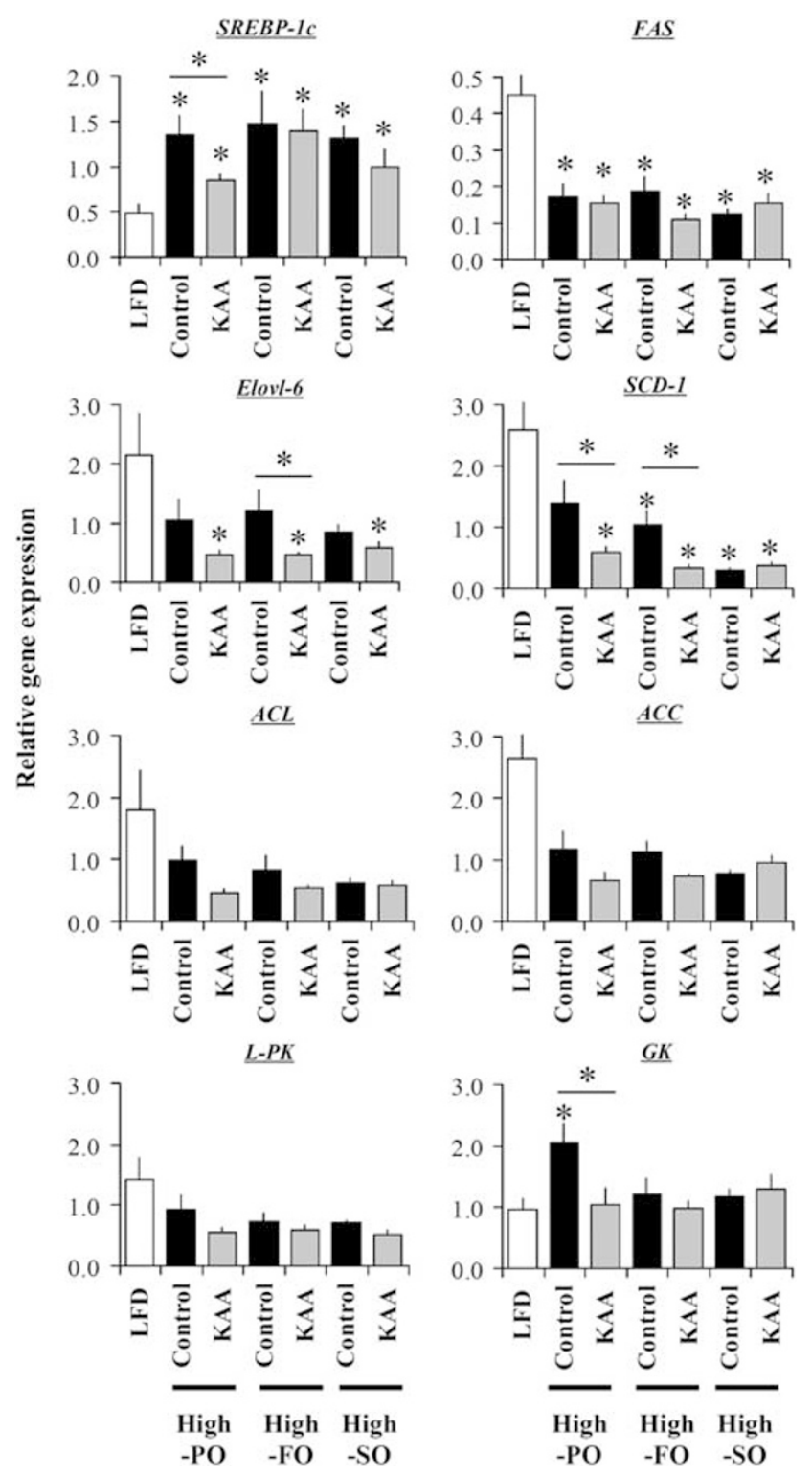

Figure 6 Changes in expression profiles of hepatic lipogenic genes under different dietary conditions. Liver samples were obtained from C57B6 mice ( $n=69$ for each group) fed for 8 weeks with an LFD or a varying type of HFD. Relative gene expression levels (normalized with $18 \mathrm{~S}$ ribosomal RNA) for SREBP-1C, fatty acid synthase (FAS), Elovl-6, stearoyl-CoA desaturase 1 (SCD1), adenosine triphosphate citrate lyase $(A C L)$, acetyl-CoA carboxylase $(A C C)$ and liver under different dietary conditions were determined using reverse transcriptase (RT)-PCR. All values are expressed as means \pm s.e.m. ( $n=6$ for each group). ${ }^{*} P<0.05$ for all HFD control groups as compared with the LFD group or as indicated.

fat accumulation by dietary KAA could be partly dependent on dietary fat content or fatty types. Our data interestingly showed that KAA supplementation exhibited a clear dependency on dietary fatty acid types in terms of the repression of fat deposition; KAA only decreased hepatic TG and cholesterol content in high-PO and high-FO diets. This difference was also observed for lipogenic gene expression. KAA supplementation of the high-PO diet decreased the expression of SREBP-1C and SCD-1, and KAA supplementation of the high-FO diet decreased the expression of SCD-1 and Elovl-6. However, KAA supplementation of the high-SO diet did not alter the expression of lipogenic genes, suggesting that these amino acids utilize some of the same factors as PUFA to regulate lipid metabolism. As compared with the high-FO diet, the high-PO diet dramatically increased hepatic $G K$ expression, and this increase was normalized by KAA supplementation. Differences in glycolytic regulation by fatty acids have been reported in vitro, where palmitate, but not oleate, directly represses GK activity or flux in liver cells. $^{29,44}$ In addition, SERBP-1c has been reported to modulate insulin-dependent GK regulation, indicating the parallel regulation of glycolysis and lipogenesis. ${ }^{45}$ The current data do not address the mechanism by which GK is regulated by dietary lipid or amino acids; thus, further study of the combined effect of fatty acids and amino acids on the regulation of hepatic glycolysis is needed.

DNL is normally assessed by profiling the expression of lipogenic genes. However, such analyses do not cover all possible regulatory mechanisms of the pathway because regulation could also occur independently of gene expression. For example, regulation can also occur through changes in substrate availability, in the phosphorylation state of the enzymes or in allosteric regulation. ${ }^{46}$ Therefore, metabolic flux analysis should be used to describe the activity or inactivity of the DNL pathway. In this study, we examined both metabolic flux analysis and gene expression. Although lipogenic gene expression was altered by dietary conditions, correlation analysis extracted a limited relationship between gene expression and actual fluxes. For instance, a positive relationship was observed between desaturase flux and SCD-1 expression. In contrast, no clear correlation was observed between elongase flux and Elovl-6 expression, although significant changes in gene expression were observed. Furthermore, the DNL flux of palmitate showed a clear correlation with $A C L$ and $A C C$ expression, but not with FAS expression. Kotronen et al. ${ }^{47}$ reported that SCD-1, but not elongase activity, was positively correlated with hepatic fat in non-alcoholic fatty liver diseased patients. The current data strongly indicate that DNL can be regulated by changes in gene expression as well as by changes in flux due to substrate availability due to dietary components, where amino acids may alter the upper pathway of lipogenesis and the desaturase fluxes.

Another important finding in this study is that tissue lipid distribution seems to be modulated by KAA supplementation, particularly in high-FO and high-SO diets. Our data show that KAA significantly reduced SO-induced hepatic and adipose accumulation of linoleate (18:2), although increased oleate (18:1) was observed in both tissues. However, KAA reduced FO-induced oleate accumulation in both tissues, but increased linoleate accumulation in adipose tissue. The mechanism underlying this exchange of tissue fatty acids 

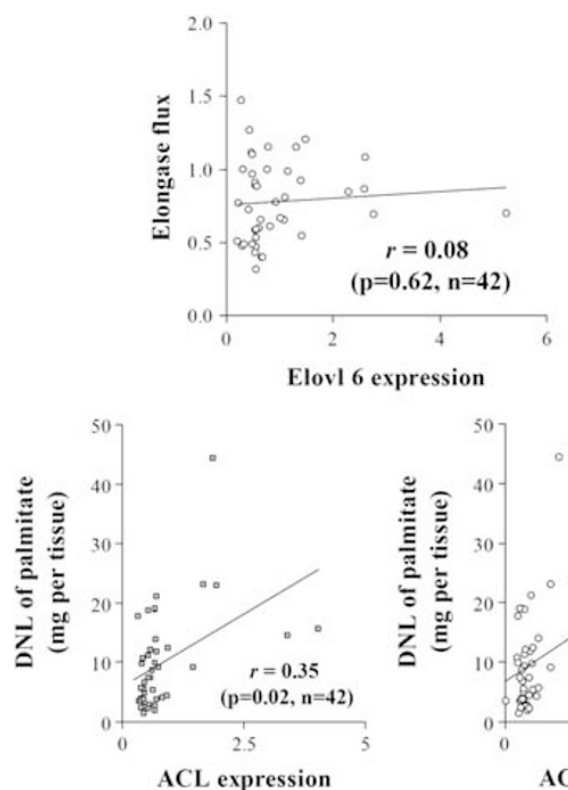
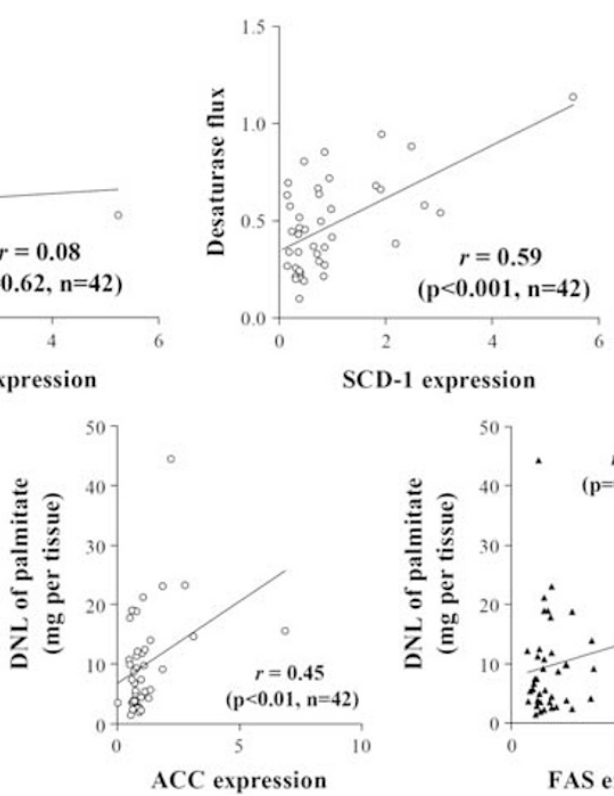

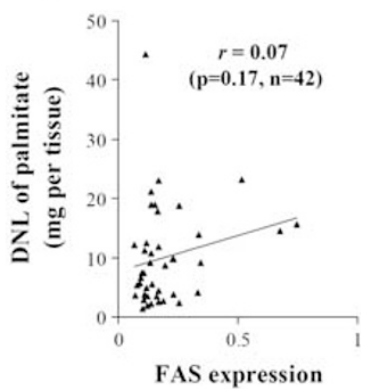

Figure 7 Correlation analysis of lipogenic fluxes and related gene expressions. DNL fluxes and gene expression were correlated. Estimations of chain elongation from palmitate $(16: 0)$ to stearate $(18: 0)$ and desaturation to oleate (18:1) were performed as described in Materials and methods section. Obtained elongase and desaturase fluxes were correlated to Elovl-6 and SCD-1 expression, respectively (upper panels). Also, ACL, ACC and FAS expressions were correlated to DNL-palmitate flux (bottom panels). Data from Figures 5 and 7 (total $n=42$ ) were used to calculate the correlation coefficients ( $r$ ).

with amino acids is unclear. However, fatty acid-binding proteins and acyl-CoA binding protein might be involved in these phenomena because these proteins determine whether lipid metabolites are used as an energy source or as signaling molecules within cells. They also regulate the circulation of lipid metabolites, which affects the tissue distribution of lipids. ${ }^{48}$ The KAA used in this study are known to generate short or branched chain acyl-CoAs that might disturb interactions with long-chain fatty acids. In addition, several studies have examined the decreased expression of intestinal fatty acid-binding protein and fatty acid transport protein- 4 induced by branched-chain amino acids in human intestinal cells. ${ }^{49}$ Of course, there may be additional factors that sense the proportion of fatty acids and amino acids in diets that regulate hepatic lipid metabolism. Thus, further metabolic profiling of amino-acid metabolism may be a possible approach to relate such metabolites to the metabolic fate of lipids and to the regulation of de novo lipid synthesis.

We studied KAA fortification in C57B6 mice after 2 months of LFD or HFD feeding. We found that KAA fortification mitigated the reduction in body fat in the preHFD-treated mice. Because we previously found that dietary KAA prevented high-fat-induced peripheral insulin resistance through the reduction of lipotoxic lipid accumulations, ${ }^{30}$ the irreversible development of peripheral insulin resistance and the subsequent impairment in substrate oxidation by long-term HFD feeding may explain it. In contrast to adipose fat, KAA fortification was effective in the reduction of hepatic fat in pre-HFD mice as well as in hyperinsulinemic $o b / o b$ mice. Thus, the regulation of hepatic lipid accumulation by KAA was independent of diet-induced insulin resistance.

In rodents, the anorexic effects of KAA loading must be considered. The accompanied decrease in caloric intake distorts metabolism as a whole, making the interpretation of experimental results difficult. Leucine, methionine and histidine have been characterized as having a strong, negative influence on food intake in rodents. ${ }^{50}$ Among these, leucine was reported to stimulate mammalian target of rapamycin signaling directly in the hypothalamus, leading to decreased food intake. ${ }^{51,52}$ In addition, aminoacid imbalance caused by supplementing either a single amino acid or a few specific amino acids negatively impacts the so-called 'metabolic value' of the diet in question. Taken together, these factors have largely confounded previous attempts to assess the direct or upstream metabolic effects of KAA. In this study, we did not observe a reduction in food intake associated with feeding a mixture of selected KAA to mice under HFD conditions, suggesting that the utilization of multiple KAA, together with dietary fat, could alleviate the potential for anorexic effects. This mixture was composed of free BCAA plus lysine and threonine, which quantitatively are the major KAA in most animal proteins. Although further optimization of our formulation is required to elucidate the contribution of each individual amino acid, the KAA diet developed in this study exhibits a marked capacity to modulate high-fat-induced metabolic alterations and to prevent subsequent pathogenesis.

In conclusion, KAA may regulate DNL through a regulatory mechanism similar to that used for PUFA, but regulation 
by KAA could also occur through pathways independent of regulation by fatty acids. In addition, the modulation of lipid partitioning could be another factor explaining the lipid-reducing effect of KAA.

\section{Conflict of interest}

All authors work at Ajinomoto Co. Inc. There is no financial support and personal connections, except for Ajinomoto Co. Inc.

\section{References}

1 Fabbrini E, Sullivan S, Klein S. Obesity and nonalcoholic fatty liver disease: biochemical, metabolic, and clinical implications. Hepatology 2010; 51: 679-689.

2 Vuppalanchi R, Chalasani N. Nonalcoholic fatty liver disease and nonalcoholic steatohepatitis: Selected practical issues in their evaluation and management. Hepatology 2009; 49: 306-317.

3 Abete I, Astrup A, Martinez JA, Thorsdottir I, Zulet MA. Obesity and the metabolic syndrome: role of different dietary macronutrient distribution patterns and specific nutritional components on weight loss and maintenance. Nutr Rev 2010; 68: 214-231.

4 Astrup A. Macronutrient balances and obesity: the role of diet and physical activity. Public Health Nutr 1999; 2: 341-347.

5 Boogaerts JR, Malone-McNeal M, Archambault-Schexnayder J, Davis RA. Dietary carbohydrate induces lipogenesis and very-lowdensity lipoprotein synthesis. Am J Physiol 1984; 246 (1 Part 1): E77-E83.

6 Chong MF, Hodson L, Bickerton AS, Roberts R, Neville M, Karpe F et al. Parallel activation of de novo lipogenesis and stearoyl-CoA desaturase activity after $3 \mathrm{~d}$ of high-carbohydrate feeding. $\mathrm{Am} \mathrm{J}$ Clin Nutr 2008; 87: 817-823.

7 Grieb P, Klapcinska B, Smol E, Pilis T, Pilis W, Sadowska-Krepa E et al. Long-term consumption of a carbohydrate-restricted diet does not induce deleterious metabolic effects. Nutr Res 2008; 28 : 825-833.

8 Bortolotti M, Kreis R, Debard C, Cariou B, Faeh D, Chetiveaux M et al. High protein intake reduces intrahepatocellular lipid deposition in humans. Am J Clin Nutr 2009; 90: 1002-1010.

9 Johnson RJ, Segal MS, Sautin Y, Nakagawa T, Feig DI, Kang DH et al. Potential role of sugar (fructose) in the epidemic of hypertension, obesity and the metabolic syndrome, diabetes, kidney disease, and cardiovascular disease. Am J Clin Nutr 2007; 86: 899-906.

10 Doucet E, Almeras N, White MD, Despres JP, Bouchard C, Tremblay A. Dietary fat composition and human adiposity. Eur J Clin Nutr 1998; 52: 2-6.

$11 \mathrm{Chu}$ SH, Samonds KW, Seronde Jr J, Hegsted DM. Protein utilization and lysine metabolism in obese and non-obese growing rats. J Nutr 1978; 108: 567-577.

12 Viviani R, Sechi AM, Lenaz G. Lipid metabolism in fatty liver of lysine- and threonine-deficient rats. J Lipid Res 1966; 7: 473-478.

13 Harbis A, Juhel C, Senft M, Lairon D. Postprandial hypertriglyceridemia induced by saturated vs. monounsaturated fatty acids is related to reduced hepatic lipoprotein receptors binding in NZW rabbits. Lipids 1999; 34 (Suppl): S125-S126.

14 Merkel M, Velez-Carrasco W, Hudgins LC, Breslow JL. Compared with saturated fatty acids, dietary monounsaturated fatty acids and carbohydrates increase atherosclerosis and VLDL cholesterol levels in LDL receptor-deficient, but not apolipoprotein E-deficient, mice. Proc Natl Acad Sci USA 2001; 98: 13294-13299.

15 Suresh Y, Das UN. Differential effect of saturated, monounsaturated, and polyunsaturated fatty acids on alloxan-induced diabetes mellitus. Prostaglandins Leukot Essent Fatty Acids 2006; 74: 199-213.

16 Chang NW, Huang PC. Comparative effects of polyunsaturatedto saturated fatty acid ratio versus polyunsaturated- and monounsaturated fatty acids to saturated fatty acid ratio on lipid metabolism in rats. Atherosclerosis 1999; 142: 185-191.

17 Rivellese AA, Maffettone A, Vessby B, Uusitupa M, Hermansen K, Berglund L et al. Effects of dietary saturated, monounsaturated and $n-3$ fatty acids on fasting lipoproteins, LDL size and postprandial lipid metabolism in healthy subjects. Atherosclerosis 2003; 167: 149-158.

18 Ghafoorunissa, Ibrahim A, Rajkumar L, Acharya V. Dietary (n-3) long chain polyunsaturated fatty acids prevent sucrose-induced insulin resistance in rats. $J$ Nutr 2005; 135: 2634-2638.

19 Lee JS, Pinnamaneni SK, Eo SJ, Cho IH, Pyo JH, Kim CK et al. Saturated, but not $n-6$ polyunsaturated, fatty acids induce insulin resistance: role of intramuscular accumulation of lipid metabolites. J Appl Physiol 2006; 100: 1467-1474.

20 Spadaro L, Magliocco O, Spampinato D, Piro S, Oliveri C, Alagona $\mathrm{C}$ et al. Effects of $\mathrm{n}-3$ polyunsaturated fatty acids in subjects with nonalcoholic fatty liver disease. Dig Liver Dis 2008; 40: 194-199.

21 Zhu FS, Liu S, Chen XM, Huang ZG, Zhang DW. Effects of n-3 polyunsaturated fatty acids from seal oils on nonalcoholic fatty liver disease associated with hyperlipidemia. World J Gastroenterol 2008; 14: 6395-6400.

22 Lavau MM, Hashim SA. Effect of medium chain triglyceride on lipogenesis and body fat in the rat. J Nutr 1978; 108: 613-620.

23 St-Onge MP, Jones PJ. Greater rise in fat oxidation with mediumchain triglyceride consumption relative to long-chain triglyceride is associated with lower initial body weight and greater loss of subcutaneous adipose tissue. Int J Obes Relat Metab Disord 2003; 27: $1565-1571$.

24 Chotechuang N, Azzout-Marniche D, Bos C, Chaumontet C, Gausseres N, Steiler T et al. mTOR, AMPK, and GCN2 coordinate the adaptation of hepatic energy metabolic pathways in response to protein intake in the rat. Am J Physiol Endocrinol Metab 2009; 297: E1313-E1323.

25 Guo F, Cavener DR. The GCN2 eIF2alpha kinase regulates fattyacid homeostasis in the liver during deprivation of an essential amino acid. Cell Metab 2007; 5: 103-114.

26 Battaile KP, Nguyen TV, Vockley J, Kim JJ. Structures of isobutyrylCoA dehydrogenase and enzyme-product complex: comparison with isovaleryl- and short-chain acyl-CoA dehydrogenases. J Biol Chem 2004; 279: 16526-16534.

27 Hostetler HA, Kier AB, Schroeder F. Very-long-chain and branched-chain fatty acyl-CoAs are high affinity ligands for the peroxisome proliferator-activated receptor alpha (PPARalpha). Biochemistry 2006; 45: 7669-7681.

28 She P, Reid TM, Bronson SK, Vary TC, Hajnal A, Lynch CJ et al. Disruption of BCATm in mice leads to increased energy expenditure associated with the activation of a futile protein turnover cycle. Cell Metab 2007; 6: 181-194.

29 Noguchi Y, Young JD, Aleman JO, Hansen ME, Kelleher JK, Stephanopoulos G. Effect of anaplerotic fluxes and amino acid availability on hepatic lipoapoptosis. J Biol Chem 2009; 284: 33425-33436.

30 Noguchi Y, Nishikata N, Shikata N, Kimura Y, Aleman JO, Young JD et al. Ketogenic essential amino acids modulate lipid synthetic pathways and prevent hepatic steatosis in mice. PLoS One 2010; 5: e12057.

31 Hara A, Radin NS. Lipid extraction of tissues with a low-toxicity solvent. Anal Biochem 1978; 90: 420-426.

32 Tserng KY, Griffin R. Quantitation and molecular species determination of diacylglycerols, phosphatidylcholines, ceramides, and sphingomyelins with gas chromatography. Anal Biochem 2003; 323: 84-93.

33 Styczynski MP, Moxley JF, Tong LV, Walther JL, Jensen KL, Stephanopoulos GN. Systematic identification of conserved 
metabolites in GC/MS data for metabolomics and biomarker discovery. Anal Chem 2007; 79: 966-973.

34 Bassilian S, Ahmed S, Lim SK, Boros LG, Mao CS, Lee WN. Loss of regulation of lipogenesis in the Zucker diabetic rat. II. Changes in stearate and oleate synthesis. Am J Physiol Endocrinol Metab 2002; 282: E507-E513.

35 Lee WN, Bassilian S, Lim S, Boros LG. Loss of regulation of lipogenesis in the Zucker diabetic (ZDF) rat. Am J Physiol Endocrinol Metab 2000; 279: E425-E432.

36 Yang D, Diraison F, Beylot M, Brunengraber DZ, Samols MA, Anderson VE et al. Assay of low deuterium enrichment of water by isotopic exchange with [U-13C3] acetone and gas chromatography-mass spectrometry. Anal Biochem 1998; 258: 315-321.

37 Moussavi N, Gavino V, Receveur O. Could the quality of dietary fat, and not just its quantity, be related to risk of obesity? Obesity (Silver Spring) 2008; 16: 7-15.

38 Piers LS, Walker KZ, Stoney RM, Soares MJ, O'Dea K. The influence of the type of dietary fat on postprandial fat oxidation rates: monounsaturated (olive oil) vs saturated fat (cream). Int J Obes Relat Metab Disord 2002; 26: 814-821.

39 Hill JO, Peters JC, Lin D, Yakubu F, Greene H, Swift L. Lipid accumulation and body fat distribution is influenced by type of dietary fat fed to rats. Int J Obes Relat Metab Disord 1993; 17: 223-236.

40 Worgall TS, Sturley SL, Seo T, Osborne TF, Deckelbaum RJ. Polyunsaturated fatty acids decrease expression of promoters with sterol regulatory elements by decreasing levels of mature sterol regulatory element-binding protein. J Biol Chem 1998; 273: 25537-25540.

41 Weigle DS, Breen PA, Matthys CC, Callahan HS, Meeuws KE, Burden VR et al. A high-protein diet induces sustained reductions in appetite, ad libitum caloric intake, and body weight despite compensatory changes in diurnal plasma leptin and ghrelin concentrations. Am J Clin Nutr 2005; 82: 41-48.

42 Noguchi Y, Shikata N, Furuhata Y, Kimura T, Takahashi M. Characterization of dietary protein-dependent amino acid metabolism by linking free amino acids with transcriptional profiles through analysis of correlation. Physiol Genomics 2008; 34: 315-326.

43 Noguchi Y, Zhang QW, Sugimoto T, Furuhata Y, Sakai R, Mori M et al. Network analysis of plasma and tissue amino acids and the

generation of an amino index for potential diagnostic use. Am J Clin Nutr 2006; 83: 513S-519S.

44 Swagell CD, Morris CP, Henly DC. Effect of fatty acids, glucose, and insulin on hepatic glucose uptake and glycolysis. Nutrition 2006; 22: 672-678.

45 Kim SY, Kim HI, Kim TH, Im SS, Park SK, Lee IK et al. SREBP-1c mediates the insulin-dependent hepatic glucokinase expression. J Biol Chem 2004; 279: 30823-30829.

46 Oosterveer MH, van Dijk TH, Tietge UJ, Boer T, Havinga R, Stellaard $\mathrm{F}$ et al. High fat feeding induces hepatic fatty acid elongation in mice. PLoS One 2009; 4: e6066.

47 Kotronen A, Seppanen-Laakso T, Westerbacka J, Kiviluoto T, Arola J, Ruskeepaa AL et al. Hepatic stearoyl-CoA desaturase (SCD)-1 activity and diacylglycerol but not ceramide concentrations are increased in the nonalcoholic human fatty liver. Diabetes 2009; 58: 203-208.

48 Storch J, Corsico B. The emerging functions and mechanisms of mammalian fatty acid-binding proteins. Annu Rev Nutr 2008; 28: 73-95.

49 Chen Q, Reimer RA. Dairy protein and leucine alter GLP-1 release and mRNA of genes involved in intestinal lipid metabolism in vitro. Nutrition 2009; 25: 340-349.

50 Peng Y, Gubin J, Harper AE, Vavich MG, Kemmerer AR. Food intake regulation: amino acid toxicity and changes in rat brain and plasma amino acids. J Nutr 1973; 103: 608-617.

51 Blouet C, Jo YH, Li X, Schwartz GJ. Mediobasal hypothalamic leucine sensing regulates food intake through activation of a hypothalamus-brainstem circuit. $J$ Neurosci 2009; 29: 8302-8311.

52 Laviano A, Meguid MM, Inui A, Rossi-Fanelli F. Role of leucine in regulating food intake. Science 2006; 313: 1236-1238; author reply $1236-8$

\section{(c)}

TMERIGHTSRESEF

Alike 3.0 Unported License. To view a copy of this license, visit http://creativecommons.org/licenses/ by-nc-sa/3.0/

Supplementary Information accompanies the paper on the Nutrition and Diabetes website (http://www.nature.com/nutd) 